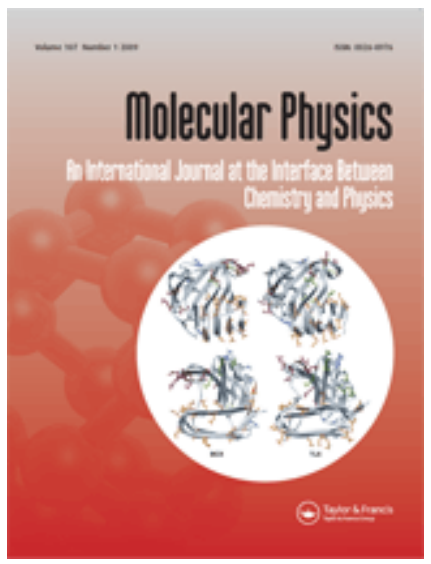

\title{
New efficient algorithm for the calculation of energy levels of AB3 type molecules
}

\begin{tabular}{|r|l|}
\hline Journal: & Molecular Physics \\
\hline Manuscript ID: & TMPH-2010-0349.R1 \\
\hline Manuscript Type: & Full Paper \\
\hline Date Submitted by the \\
Author: & $29-$ Sep-2010 \\
\hline Complete List of Authors: & Nikitin, Andrei; GSMA, university of Reims \\
\hline Keywords: & $\begin{array}{l}\text { symmetric-top molecules, variational calculation, irreducible tensor, } \\
\text { finite basis representation, vibration energy levels }\end{array}$ \\
\hline &
\end{tabular}

\section{SCHOLARONE ${ }^{m}$ Manuscripts}




\title{
New efficient algorithm for the calculation of energy levels of $\mathbf{A B}_{3}$ type molecules
}

\author{
Andrei V. Nikitin \\ Groupe de Spectrométrie Moléculaire et Atmosphérique, UMR CNRS 6089, Université de \\ Reims, U.F.R. Sciences, B.P. 1039, 51687 Reims Cedex 2, France \\ and \\ Laboratory of Theoretical Spectroscopy, Institute of Atmospheric Optics, Russian Academy of \\ Science, 634055 Tomsk, Russia
}

Keywords: symmetric-top molecules, variational calculation, irreducible tensor, finite basis representation, vibration energy levels

Current correspondence address:

Dr. Andrei V. Nikitin, GSMA, Université de Reims, U.F.R. Sciences, B.P. 1039, 51687 Reims Cedex 2, France

Phone: +33-326913380,

E-mail: avn@1ts.iao.ru, andreinikitin076@mail.ru 


\begin{abstract}
This paper illustrates a method for the construction of a symmetry-adapted contracted angular basis set for $\mathrm{AB}_{3}$ molecules. Simple formulas that use this basis set for calculations of the angular matrix elements of the kinetic energy (KE) operator and angular matrix elements of potential energy surface (PES) are reported. The efficient recursive algorithm based on the tensorial formalism is used for the calculation of vibrational matrix elements. The symmetric form of vibration $\mathrm{KE}$ operators without the $\sin (q)^{-2}$ type singularity is applied. A good basis set convergence for the calculations of vibrational levels of the $\mathrm{PH}_{3}$ molecule is demonstrated.
\end{abstract}




\section{INTRODUCTION}

The theory and computational modeling of molecular vibrations is on the top of interest in the molecular spectroscopy and related fields. Advances in computation of vibration-rotation spectra of three-atomic molecules (see ref. 1-3 and references therein) resulted in an interest to computations of spectra for polyatomic molecules [4-31]. For more information on this subject, the reader is referred to the recent review [21]. An important part of polyatomic molecules are high symmetry molecules [4-14,18-27]. For the $\mathrm{ABC}_{3}$ type molecules variational calculations using a finite basis representations and full $C_{3 v}$ symmetry have been applied to $\mathrm{CH}_{3} \mathrm{~F}[10,11]$ and $\mathrm{CH}_{3} \mathrm{Cl}$ [27] molecules. In this paper $C_{3 v}$ and $T_{d}$ point groups are considered to be isomorphic to | finite permutation groups (permutations of three or four atoms). In spite of a considerable number of publications devoted to computation of vibration-rotation spectra of the $A_{4}$ and the $A B_{3}$ type molecules, they often do not completely use the molecular symmetry $[4,5,8,9,19,25,26]$. One variational method for calculating excited bending states of symmetric tetrahedral pentaatomic molecules based on the use of Radau coordinates and Jacobi polynomials as the basis functions has been presented in [30,31]. Only the technique based on the normal or symmetry coordinates does not result in special difficulties related to a construction of the symmetry basis [13,17,18,21]. See also rovibration calculations by Rey et al. [24] using rectilinear normal coordinates and the normal mode Hamiltonian expressed in terms of irreducible tensor operators.

When using the internal coordinates, such as three angles between the H-P-H bonds in $\mathrm{PH}_{3}$, in the general case, the basis functions constructed on the base of these coordinates are not orthogonal, even though those angles are independent. The construction of the symmetry basis using the $q_{i j}$ angles is a trivial task, but a problem is to work out an effective technique for calculations of multidimensional integrals using this basis. In the present work, a finite basis representations approach was applied for the $\mathrm{AB}_{3}$ type molecules and simple formulas for calculations of the matrix elements of the KE operator and PES are reported. As a rule, we will consider only the angular part of the matrix elements, because the symmetry properties of radial part are obvious. Here we also assume that the molecule has only one deep minimum.

Tennyson et al.[32], Gatti et al. [33,34], Mladenovic' [35-3ㅁ] have presented derivations of kinetic energy operators for treating rotations and vibrations of polyatomic molecules using polyspherical coordinates. In these coordinates, the $N$ nuclear position vectors are transformed to 
$N-1$ internal vectors and the nuclear center of mass vector, and the internal vectors are parametrized by spherical polar coordinates: $R_{i}, \theta_{i}, \varphi_{i}, i=1, \ldots, N-1$. Then, a body-fixed coordinate system is introduced, defined by aligning the body-fixed $z$ axis along the first vector, and placing the second vector in the body-fixed $x z$ plane. The remaining angles $\theta_{i}, \varphi_{i}$ are either referenced with respect to the body-fixed $z$ axis, or other vectors.

At the same time, the symmetric form of vibration $\mathrm{KE}$ operators is known [30,31,37,3$\underline{8}]$. Instead of using the torsion angle, symmetric form use additional angles between molecular bonds. This form of KE operators is more tailored to our basis functions, and so we use it in the present work. Symmetric form is especially interesting because it has no $\sin (\mathrm{q})^{-2}$ singularity while the KE operator of Ref. [35] contains this singularity. When constructing the vibration basis set using the products of functions that depend on the bending $q$ and torsion $t$ angles, the angular basis $f_{n}(q)$ must be chosen to be vanishing at $\sin (q)=0$. From a physical point of view, this choice of $f_{n}(q)$ is not always correct because in some vibration states, a molecule can be in a geometric configuration with the angle $q=\pi$ with rather high probability.

Described in this work algorithm of calculation of vibrational energy levels uses nonorthogonal basis set of three interbond angles $\mathrm{q}_{\mathrm{ij}}$. Of course, the non-orthogonal basis is not always easy-to-use, but this type of basis is used, for example, in the chemical reactions theory [39]. When calculating the matrix elements of PES, whenever possible, we try to keep the symmetrical form with respect to three angles. We transform to polar coordinates only at the final phase of the calculations. Similarly, when calculating the matrix elements of the KE operator in symmetric form, we first calculate the derivatives with respect to angles using matrix element symmetry, and only after this, we calculate the matrix element by using polar coordinates.

The present paper comprises seven sections. Section II describes an algorithm for constructing contracted angular basis set that uses three angles $\mathrm{q}_{\mathrm{ij}}$ between three identical atoms, and the norm calculation for this basis set. Sections III and IV are devoted to algorithm of 


\section{CONTRACTED ANGULAR BASIS FOR THREE IDENTICAL ATOMS AND NORM}

\section{CALCULATION}

In the present work, the following coordinates were chosen as independent ones: three bond lengths $\left\{r_{1}, r_{2}, r_{3}\right\}$ and three interbond angles $\left\{q_{12}, q_{13}, q_{23}\right\}$. For one-dimensional basis functions, the following functions were used: $f_{n}(q)=\sqrt{2 \pi} \sum_{l} c_{n l} Y_{l, s}(q, 0)$, where $Y$ are spherical harmonics written in the form of Ref. [40] that ensures that $Y_{0,0}(q, 0)=\frac{1}{\sqrt{4 \pi}}$ and $2 \pi \int_{0}^{\pi} Y_{l, s}^{2}(q, 0) d \cos (q)=1$.

The normalized eigenfunctions $\int_{0}^{\pi} f_{n}^{2}\left(q_{23}\right) d \cos \left(q_{23}\right)=1$ of one-dimensional angular problem can be used to obtain $f_{n}$. Initial angular basis can be written in the form:

$$
\left|n_{1} n_{2} n_{3}\right\rangle=f_{n_{1}}\left(q_{12}\right) f_{n_{2}}\left(q_{13}\right) f_{n_{3}}\left(q_{23}\right) .
$$

This basis is not orthogonal one. The norm is calculated using the following three-dimensional integral:

$$
<n_{1} n_{2} n_{3} \mid k_{1} k_{2} k_{3}>=\int_{0}^{\pi} \int_{0}^{\pi} \int_{0}^{2 \pi} f_{n_{1}}\left(q_{12}\right) f_{k_{1}}\left(q_{12}\right) f_{n_{2}}\left(q_{13}\right) f_{k_{2}}\left(q_{13}\right) f_{n_{3}}\left(q_{23}\right) f_{k_{3}}\left(q_{23}\right) d \cos \left(q_{12}\right) d \cos \left(q_{13}\right) d t_{23}
$$

where $\mathrm{t}_{23}$ is the torsion angle.

To calculate the integral $\int_{0}^{2 \pi} f_{n}^{*}\left(q_{23}\right) f_{m}\left(q_{23}\right) d t_{23}=2 \pi \int_{0}^{2 \pi} \sum_{l_{1}, l_{2}} c_{n l_{1}} c_{m l_{2}} Y_{l_{1}, s}^{*}\left(q_{23}, s\right) Y_{l_{2}, s}\left(q_{23}, s\right) d t_{23}$ the following expressions [40] were used:

$Y_{l_{1} s_{1}}\left(q_{23}, 0\right) Y_{l_{2} s_{2}}\left(q_{23}, 0\right)=\sum_{L M} \sqrt{\frac{\left(2 l_{1}+1\right)\left(2 l_{2}+1\right)}{4 \pi(2 L+1)}} C\left(l_{1} 0, l_{2} 0, L 0\right) C\left(l_{1} s_{1}, l_{2} s_{2}, L M\right) Y_{L M}\left(q_{23}, 0\right)$ 
and

$$
Y_{l, 0}\left(\cos \left(q_{12}\right) \cos \left(q_{13}\right)+\sin \left(q_{12}\right) \sin \left(q_{13}\right) \cos \left(t_{23}\right)\right)=\sqrt{\frac{4 \pi}{2 l+1}} \sum_{m} Y_{l m}\left(q_{12}, 0\right) Y_{l m}\left(q_{13}, t_{23}\right)
$$

Therefore, from Eqs. $(2,3)$, we get:

$$
Y_{l_{1} 0}\left(q_{23}, 0\right) Y_{l_{2} 0}\left(q_{23}, 0\right)=\sum_{L} K_{l_{1} l_{2} L} \sum_{m} Y_{L m}\left(q_{12}, 0\right) Y_{L m}\left(q_{13}, t_{23}\right)
$$

where $K_{l_{1} l_{2} L}=\frac{\sqrt{\left(2 l_{1}+1\right)\left(2 l_{2}+1\right)}}{2 L+1} C\left(l_{1} 0, l_{2} 0, L 0\right)^{2}, \quad$ and $\quad C\left(l_{1} m_{1}, l_{2} m_{2}, L M\right)$ is Clebsch-Gordan $\begin{array}{lllll}\text { coefficient. Using } & \text { Eq. } & \text { (4) }\end{array}$

$$
\begin{aligned}
& <n_{1} n_{2} n_{3} \mid k_{1} k_{2} k_{3}>=\int_{0}^{\pi} \int_{0}^{\pi} f_{n_{1}}\left(q_{12}\right) f_{k_{1}}\left(q_{12}\right) f_{n_{2}}\left(q_{13}\right) f_{k_{2}}\left(q_{13}\right) \\
& (2 \pi)^{2} \sum_{l_{1} l_{2}} c_{n_{3} l_{1}} c_{k_{3} l_{2}} \sum_{L} K_{l_{1} l_{L} L} Y_{L 0}\left(q_{12}, 0\right) Y_{L 0}\left(q_{13}, 0\right) d \cos \left(q_{12}\right) d \cos \left(q_{13}\right)
\end{aligned}
$$

Let us denote the one-dimensional integral by

$J_{n_{1}, k_{1}, L, v}=\int_{0}^{\pi} f_{n_{1}}\left(q_{12}\right) f_{k_{1}}\left(q_{12}\right) Y_{L v}\left(q_{12}, 0\right) d \cos \left(q_{12}\right)=2 \pi \sum_{l_{1} l_{2}} c_{n_{1} l_{1}} c_{k_{1} l_{2}} \int_{0}^{\pi} Y_{l_{1} 0}\left(q_{12}, 0\right) Y_{l_{2} 0}\left(q_{12}, 0\right) Y_{L v}\left(q_{12}, 0\right) d \cos \left(q_{12}\right)$

Using Eq. (2) and taking into account that spherical harmonics $Y_{l m}$ with the same $m$ are orthogonal we

get:

$$
J_{n_{1}, k_{1}, L, 0}=\sum_{l_{1} l_{2}} c_{n_{1} l_{1}} c_{k_{1} l_{2}} \sqrt{\frac{\left(2 l_{1}+1\right)\left(2 l_{2}+1\right)}{4 \pi(2 L+1)}} C\left(l_{1} 0, l_{2} 0, L 0\right)^{2}=\frac{1}{\sqrt{4 \pi}} \sqrt{(2 L+1)} \sum_{l_{1} l_{2}} c_{n_{1} l_{1}} c_{k_{1} l_{2}} K_{l_{1}, l_{2}, L}
$$

Using Eqs. $(5,6)$, one can derive

$$
<n_{1} n_{2} n_{3} \mid k_{1} k_{2} k_{3}>=(2 \pi)^{3} \sum_{L} \frac{1}{\sqrt{\pi(2 L+1)}} J_{n_{1}, k_{1}, L 0} J_{n_{2}, k_{2}, L 0} J_{n_{3}, k_{3}, L 0}
$$

For example, if $c_{n l}=\delta_{n l}$ we get: $<000|000>=\pi,<111| 111>=\frac{33 \pi}{25},<112 \mid 112>=\frac{43 \pi}{35}$.

III. CALCULATION OF MATRIX ELEMENTS OF PES FOR THREE IDENTICAL ATOMS 
For majority of molecules, the angular PES can be expressed in terms of a sum of product functions: $\cos ^{P_{12}}\left(q_{12}\right) \cos ^{P_{13}}\left(q_{13}\right) \cos ^{P_{23}}\left(q_{23}\right)$. Due to simple recursive formulas [40] for $\cos (q) Y_{n, 0}(q)$, this considerably facilitates the calculation of matrix elements. Therefore,

$$
\cos ^{P}(q) f_{k}(q)=\sqrt{2 \pi} \sum_{l_{2} l_{3}} c_{k l_{2}}<l_{3} s\left|\cos ^{P}(q)\right| l_{2} s>Y_{l_{3}, s}(q, 0)
$$

If $s=0$, similarly to Eqs. $(5,6)$, we get the following expression for the matrix element:

$$
\begin{aligned}
< & n_{1} n_{2} n_{3}\left|\cos \left(q_{23}\right)^{P}\right| k_{1} k_{2} k_{3}>=(2 \pi)^{2} \int_{0}^{\pi} \int_{0}^{\pi} f_{n_{1}}\left(q_{12}\right) f_{k_{1}}\left(q_{12}\right) f_{n_{2}}\left(q_{13}\right) f_{k_{2}}\left(q_{13}\right) \\
& \sum_{l_{1} l_{2} l_{3}} c_{n_{3} l_{1}} c_{k_{3} l_{2}}<l_{3} 0\left|\cos ^{P}\right| l_{2} 0>\sum_{L} K_{l_{1} l_{3} L} Y_{L 0}\left(q_{12}, 0\right) Y_{L 0}\left(q_{13}, 0\right) d \cos \left(q_{12}\right) d \cos \left(q_{13}\right)
\end{aligned}
$$

In comparison with Eq. (5), this expression contains an additional summation over the $l_{3}$ index and the $<l_{3}\left|\cos ^{p}\right| l_{2}>$ matrix element. Using the expression

$$
J_{n_{3}, k_{3}, L, 0}^{P}=2 \pi \sum_{l_{1} l_{2} l_{3}} c_{n_{3} l_{1}} c_{k_{3} l_{2}}<l_{3} 0\left|\cos ^{P}\right| l_{2} 0>K_{l_{1} l_{3} L} \sqrt{\frac{2 L+1}{4 \pi}}
$$

one can write the matrix element in the form:

$$
\begin{gathered}
<n_{1} n_{2} n_{3}\left|\cos \left(q_{23}\right)^{P}\right| k_{1} k_{2} k_{3}>=(2 \pi)^{2} \sum_{l_{1} l_{3} l_{3}} c_{n_{3} l_{1}} c_{k_{3} l_{2}}<l_{3} 0\left|\cos ^{P}\right| l_{2} 0>\sum_{L} K_{l_{1} l_{3} L} J_{n_{1}, k_{1}, L 0} J_{n_{2}, k_{2}, L 0}= \\
(2 \pi)^{3} \sum_{L} \frac{1}{\sqrt{\pi(2 L+1)}} J_{n_{1}, k_{1}, L 0}^{P} J_{n_{2}, k_{2}, L 0} J_{n_{3}, k_{3}, L 0}
\end{gathered}
$$

Taking into account that $q_{23}$ is independent of $q_{12}$ and $q_{13}$, it is easy to obtain the following general expression for the PES matrix elements:

$$
<n_{1} n_{2} n_{3}\left|\cos \left(q_{12}\right)^{P_{12}} \cos \left(q_{13}\right)^{P_{13}} \cos \left(q_{23}\right)^{P_{23}}\right| k_{1} k_{2} k_{3}>=(2 \pi)^{3} \sum_{L} \frac{1}{\sqrt{\pi(2 L+1)}} J_{n_{1}, k_{1}, L 0}^{P_{12}} J_{n_{2}, k_{2}, L 0}^{P_{P}} J_{n_{3}, k_{3}, L 0}^{P_{P_{3}}}
$$

\section{CALCULATION OF MATRIX ELEMENTS OF KE}

In this section, we use the mass-dependent orthogonal coordinates $\left\{r_{1}^{\prime}, r_{2}^{\prime}, r_{3}^{\prime}, q_{12}^{\prime}, q_{13}^{\prime}, q_{23}^{\prime}\right\}$. The polar coordinates are defined in a standard way via three vectors $\left\{\vec{r}_{i}\right\}(i=1,2,3)$ linking the centre of mass with three identical atoms. Three stretching coordinates $r_{i}$ are the lengths of these vectors 
and $\left\{q_{i j}\right\}$ are interbond angular coordinates. The mass-dependent orthogonal coordinates [3 $\underline{5}$ ] $\left\{r_{1}^{\prime}, r_{2}^{\prime}, r_{3}^{\prime}, q_{12}^{\prime}, q_{13}^{\prime}, q_{23}^{\prime}\right\}$ are defined in a similar way via three vectors $\left\{\vec{r}_{i}^{\prime}\right\}$ :

$$
\vec{r}_{i}^{\prime}=\vec{r}_{i}+d \sum_{j=1}^{3} \vec{r}_{j}, \text { where } d=-\frac{1}{3}+\frac{1}{3 \sqrt{1-3 \mu_{c}}} \text { with } \mu_{c}=\frac{m_{H}}{3 m_{H}+m_{P}}
$$

The most convenient expression for the kinetic energy can be obtained in terms of these coordinates, while the analytical representation using internal polar coordinates includes additional terms in the kinetic energy operator [3ㄷ]. Mass-dependent coordinates (10) keep the same symmetry properties as initial polar coordinates. Let us use the KE operator in the form Ref. $\left[3 \underline{0,31,37]}\right.$ where the KE operator does not comprise the singular terms of the $\sin (q)^{-2}$ form. Therefore one can use the $Y_{n, 0}(q, 0)$ spherical harmonics as basis functions that do not vanish at $q=\pi$. The kinetic energy of the $\mathrm{AB}_{4}$ - and $\mathrm{AB}_{3}$-type molecules can be expressed in the form [37]:

$$
T_{V} /\left(-\frac{1}{2} h^{2}\right)=\sum_{j k}^{3 N-6} g^{j k} \frac{\partial^{2}}{\partial \Re_{j} \partial R_{k}}+\sum_{j}^{3 N-6} h^{j} \frac{\partial}{\partial R_{j}} .
$$

For radial coefficients $g^{i j}=\frac{\delta_{i j}}{m_{i}}$. The angular diagonal coefficients of the $g$ matrix are written in the form: $\sin ^{2}\left(q_{i j}\right)\left(\frac{1}{m_{i} r_{i}^{2}}+\frac{1}{m_{j} r_{j}^{2}}\right) \frac{\partial^{2}}{\partial^{2} \cos \left(q_{i j}\right)}$. For a pair of angles $q_{i j} q_{l k}$, the angular off-diagonal elements of the $g$ matrix vanish unless $i=l$ or $j=k$ : $g^{\cos \left(q_{i j}\right), \cos \left(q_{j k}\right)}=\frac{-\cos \left(q_{i j}\right) \cos \left(q_{j k}\right)+\cos \left(q_{i k}\right)}{m_{j} r_{j}^{2}} \frac{\partial^{2}}{\partial \cos \left(q_{i j}\right) \partial \cos \left(q_{j k}\right)}$. Note, that in the $g$ matrix, all off-diagonal radial-angular elements vanish. The radial and angular coefficients of the $h$ matrix are correspondingly $h^{i}=\frac{2}{m_{i} r_{i}}$ and $h^{\cos \left(q_{i j}\right)}=-2 \cos \left(q_{i j}\right)\left(\frac{1}{m_{i} r_{i}^{2}}+\frac{1}{m_{j} r_{j}^{2}}\right) \frac{\partial}{\partial \cos \left(q_{i j}\right)}$. For $\mathrm{AB}_{4}$ molecules, the summation in (11) is performed over four radial coordinates $r_{i}$ and six angles $q_{i j}$, while for $\mathrm{AB}_{3}$ molecules, only three radial coordinates $r_{i}$ and three angles $q_{i j}$ are used. In this 
paper, the sums over the diagonal and angular first derivative $h^{i} \frac{\partial}{\partial \mathfrak{R}_{i}}$ terms are referred to as a diagonal term. It is easy to calculate the diagonal matrix element using the following equality [40]: $\left(\frac{\partial^{2}}{\partial^{2} q_{i j}}+\operatorname{ctg}\left(q_{i j}\right) \frac{\partial}{\partial q_{i j}}\right) Y_{l, 0}\left(q_{i j}, 0\right)=-l(l+1) Y_{l, 0}\left(q_{i j}, 0\right)$.

Note that only the full sum of all diagonal and off-diagonal angular terms results in symmetric matrix elements, while each of these sums, e.g. the summation only over the diagonal or offdiagonal terms, results in asymmetric matrix elements. In the present paper, to calculate matrix elements, we use the following property of the reduced matrix elements_[41,42]: $<F_{L}|\hat{O}| F_{R}>=<P F_{L}|P \hat{O}| P F_{R}>$ where $P$-is an element of the molecular symmetry group. Due to this property, for $A B_{3}$ molecules, it is not mandatory to calculate all three diagonal and offdiagonal angular matrix elements. It is sufficient to calculate only one simplest diagonal and one off-diagonal angular matrix element. We calculate the matrix element for the sum of three diagonal operators using the expression:

$$
\begin{gathered}
\left\langle n_{1} n_{2} n_{3}\left|\frac{\partial^{2}}{\partial q_{23}^{2}}+\operatorname{ctg}\left(q_{23}\right) \frac{\partial}{\partial q_{23}}\right| k_{1} k_{2} k_{3}\right\rangle=(2 \pi)^{3} \int_{0}^{\pi} \int_{0}^{\pi} \int_{0}^{2 \pi} \sum_{l_{1} l_{2}^{\prime} l_{2} l_{2} l_{2} l_{n_{3}^{\prime}}{ }_{n_{1}} c_{1}} c_{k_{1} l_{1}^{\prime}} c_{n_{2} l_{2}} c_{k_{2} l_{2}} c_{n_{3} l_{3}} c_{k_{3} l_{3}}\left\{l_{3}^{\prime}\left(l_{3}^{\prime}+1\right)\right\} \\
Y_{l_{1}, 0}^{*}\left(q_{12}, 0\right) Y_{l_{1} 0}\left(q_{12}, 0\right) Y_{l_{2}, 0}^{*}\left(q_{13}, 0\right) Y_{l_{2} 0}\left(q_{13}, 0\right) Y_{l_{3}, 0}^{*}\left(q_{23}, 0\right) Y_{l_{3} 0}\left(q_{23}, 0\right) d \cos \left(q_{12}\right) d \cos \left(q_{13}\right) d t_{23}
\end{gathered}
$$

Using the designation

$$
J_{n_{3}, k_{3}, L, 0}^{D 2}=\sum_{l_{3} l_{3}^{\prime}} c_{n_{3} l_{3}} c_{k_{3} l_{3}^{\prime}}\left[l_{3}^{\prime}\left(l_{3}^{\prime}+1\right)\right] K_{l_{3} l^{\prime} L} \sqrt{\frac{2 L+1}{4 \pi}},
$$

we can write the diagonal matrix element it the following form:

$$
\left\langle n_{1} n_{2} n_{3}\left|\frac{\partial^{2}}{\partial q_{23}^{2}}+\operatorname{ctg}\left(q_{23}\right) \frac{\partial}{\partial q_{23}}\right| k_{1} k_{2} k_{3}\right\rangle=(2 \pi)^{3} \sum_{L} \frac{1}{\sqrt{\pi(2 L+1)}} J_{n_{1}, k_{1}, L 0}^{0} J_{n_{2}, k_{2}, L 0}^{0} J_{n_{3}, k_{3}, L 0}^{D 2}
$$

It is more convenient to calculate the off-diagonal matrix element using the polar coordinates. Let us show, that the off-diagonal matrix element can be expressed in the following form: $\left\langle n_{1} n_{2} n_{3}\left|\cos \left(t_{23}\right) \frac{\partial}{\partial q_{12}} \frac{\partial}{\partial q_{13}}\right| k_{1} k_{2} k_{3}\right\rangle=(2 \pi)^{3} \sum_{L} J_{n_{1}, k_{1}, L, 1}^{D 1} J_{n_{2}, k_{2}, L, 1}^{D 1} J_{n_{3}, k_{3}, L, 0} \frac{1}{\sqrt{\pi(2 L+1)}}$,

where $J_{n_{1} k_{1}, 1,1}^{D 1}$ is a coefficient similar to the coefficients used in Eqs. (6), (8), (12). 
To prove the formula (14), we use Eq. (4) for product of $\mathrm{J}$ functions depending on $q_{23}$, integrate Eq. (14) over $t_{23}$, and using the known formula for spherical harmonics [40] $Y_{l,-m}(q, \varphi)=(-1)^{m} Y_{l, m}(q,-\varphi)$, we get:

$$
\begin{aligned}
& \int_{0}^{\pi} \int_{0}^{\pi} \int_{0}^{2 \pi} f_{n_{1}}\left(q_{12}\right) f_{n_{2}}\left(q_{13}\right) f_{n_{3}}\left(q_{23}\right)\left(\cos \left(t_{23}\right) \frac{\partial}{\partial q_{12}} \frac{\partial}{\partial q_{13}}\right) f_{k_{1}}\left(q_{12}\right) f_{k_{2}}\left(q_{13}\right) f_{k_{3}}\left(q_{23}\right) d \cos \left(q_{12}\right) d \cos \left(q_{13}\right) d t_{23}=(2 \pi)^{2} \times \\
& \sum_{l_{1} l_{2}} c_{n_{3} l_{1}} c_{k_{3} l_{2}} \int_{0}^{\pi} \int_{0}^{\pi} f_{n_{1}}\left(q_{12}\right) f_{n_{2}}\left(q_{13}\right) \sum_{L} K_{l_{1} L}\left(\frac{\partial}{\partial q_{12}} \frac{\partial}{\partial q_{13}} f_{k_{1}}\left(q_{12}\right) f_{k_{2}}\left(q_{13}\right)\right) Y_{L, 1}\left(q_{12}, 0\right) Y_{L, 1}\left(q_{13}, 0\right) d \cos \left(q_{12}\right) d \cos \left(q_{13}\right)
\end{aligned}
$$

If we denote the one-dimensional integrals in the right-hand member of this expression via $J_{n_{1} k_{1} L, 1}^{D 1}=\int f_{n_{1}}\left(q_{12}\right)\left(\frac{\partial}{\partial q_{12}} f_{k_{1}}\left(q_{12}\right)\right) Y_{L, 1}\left(q_{12}, 0\right) d \cos \left(q_{12}\right)$, we get Eq. (14)

One can simplify the expression for the $J_{n_{1} k_{1} L, 1}^{D 1}$ coefficient using the following formula [40]: $\frac{\partial}{\partial q} Y_{l, m}(q)=\operatorname{mctg}(q) Y_{l, m}(q)+\sqrt{l(l+1)-m(m+1)} Y_{l, m+1}(q)$, we get: $J_{n_{1} k_{1} L, 1}^{D 1}=(2 \pi) \sum_{l_{1} l_{2}} c_{k_{1} l_{1}} c_{k_{2} l_{2}} \int Y_{l_{1} 0}\left(q_{12}, 0\right) \sqrt{l_{2}\left(l_{2}+1\right)} Y_{l_{2} 1}\left(q_{12}, 0\right) Y_{L, 1}\left(q_{12}, 0\right) d \cos \left(q_{12}\right)$. Using Eq. (2) with $\mathrm{s}_{1}=0, \mathrm{~s}_{2}=1$, and taking into account the orthogonality of spherical harmonics, we get

$$
J_{n_{1} k_{1} L, 1}^{D 1}=\sum_{l_{1} l_{2}} c_{k_{1} l_{1}} c_{k_{2} l_{2}} \sqrt{l_{2}\left(l_{2}+1\right)} \sqrt{\frac{\left(2 l_{1}+1\right)\left(2 l_{2}+1\right)}{4 \pi(2 L+1)}} C\left(l_{1}, 0, l_{2}, 0, L, 0\right) C\left(l_{1}, 0, l_{2}, 1, L, 1\right)
$$

For even values of $l_{1}+l_{2}+L$, it can be shown [40] that:

$$
C\left(l_{1}, 0, l_{2}, 1, L, 1\right)=C\left(l_{1}, 0, l_{2}, 0, L, 0\right) \frac{l_{2}\left(l_{2}+1\right)+L(L+1)-l_{1}\left(l_{1}+1\right)}{2\left[L(L+1) l_{2}\left(l_{2}+1\right)\right]^{1 / 2}}
$$

Since the $C\left(l_{1}, 0, l_{2}, 0, L, 0\right)$ coefficients are not equal to zero only at even values of $l_{1}+l_{2}+L$, one can simplify the expression obtained for $\mathrm{J}$ in the following way:

$$
J_{n_{1} k_{1} L, 1}^{D 1}=\sum_{l_{1} l_{2}} c_{k_{1} l_{1}} c_{k_{2} l_{2}} K_{l_{1} L} \frac{l_{2}\left(l_{2}+1\right)+L(L+1)-l_{1}\left(l_{1}+1\right)}{4[\pi L(L+1)]^{1 / 2}}[2 L+1]^{1 / 2}
$$

\section{FULL SYMMETRY BASIS AND CALCULATION OF VIBRATIONAL LEVELS}

The initial angular basis (1) can be easily symmetrized and tailored for calculations that use a symmetrical basis. Using the standard operators, one can construct the full symmetry basis set of functions:

$$
\left.\left|n_{1} n_{2} \ldots n_{S}\right| k G \sigma\right\rangle=\hat{P}_{k G \sigma}\left|n_{1} n_{2} \ldots n_{S}\right\rangle
$$


For example, for $\mathrm{AB}_{3}$ molecules, we use the following six projection operators (17) to construct symmetrized three-dimensional angular $\left|n_{12} n_{13} n_{23}, k_{A} G_{A} \sigma_{A}\right\rangle$ and radial $\left|n_{1} n_{2} n_{3}, k_{R} G_{R} \sigma_{R}\right\rangle$ basis sets:

$$
\begin{aligned}
& P_{A_{1}}=\left(e+(12+(13))(e+(23)) / \sqrt{6}, \quad P_{A_{2}}=(e-(12)-(13))(e-(23)) / \sqrt{6},\right. \\
& P_{1 E_{a}}=((13)+(12)-2 e)(e+(23)) / \sqrt{12}, \quad P_{1 E_{b}}=((13)-(12))(e+(23)) / 2, \\
& P_{2 E_{a}}=((13)-(12))(e-(23)) / 2, \quad P_{2 E_{b}}=(-(12)-(13)-2 e)(e-(23)) / \sqrt{12}
\end{aligned}
$$

Note that in the present work, the angular $\left|n_{12} n_{13} n_{23}, k_{A} G_{A} \sigma_{A}\right\rangle$ and radial $\left|n_{1} n_{2} n_{3}, k_{R} G_{R} \sigma_{R}\right\rangle$ basis sets also are referred to as $f_{3 D, B A B}^{G}\left(q_{12}, q_{13}, q_{23}\right)$ and $f_{3 D, A B}^{G}\left(r_{1}, r_{2}, r_{3}\right)$, respectively. The vibrational basis functions and the PES operators are expressed in terms of irreducible tensor operators [41,42]. Each tensor is associated to a binary tree [4ㄹ] . The binary trees of vibrational coupling for $\mathrm{AB}_{3}$ molecules are shown in Fig.1. Each branch of the tree is characterized by its symmetry. The binary tree of the PES parameters is shown in the left part of the figure while the binary tree of the vibrational basis coupling is shown in the right part of this figure. In order to determine the PES operators of the $A_{1}$ symmetry type, formed from the symmetrized coordinates_[24,25] $S R_{A_{1}}, S R_{E a}, S R_{E b}, S A_{A_{1}}, S A_{E_{a}}, S A_{E_{b}}$ we used the two step procedure. In first step, we construct the symmetrized power of the coordinates $\left[S R_{A_{1}}\right]^{P_{1}},\left[S R_{E}\right]^{P_{2}},\left[S A_{A_{1}}\right]^{P_{3}},\left[S A_{E}\right]^{P_{4}}$. In second step, we couple the symmetrized powers of different symmetrized coordinates into irreducible trees. A set of all possible trees of the $A_{1}$ symmetry type gives a final set of trees. The potential function was developed in power series of irreducible tensors $V\left(r_{1}, \ldots r_{n}\right)=\sum_{i} K_{i} R_{i}\left(r_{1}, \ldots r_{n}\right)$. The complete vibrational basis functions can be constructed by coupling the symmetrized basis functions of all coordinates into binary trees. For $\mathrm{AB}_{3}$ and $\mathrm{AB}_{4}$ molecules [2므,27], it is necessary to perform only one coupling of stretching basis function and bending basis function $\left(\left|n_{12} n_{13} n_{23}, G_{A}\right\rangle\right.$ and 
$\left|n_{1} n_{2} n_{3}, G_{R}\right\rangle$ in Fig 1). We used the following standard definition of the direct product of irreducible tensors $[\underline{41}, 4 \underline{2}]$ :

$\left(T^{C^{\prime}} \times T^{C^{\prime \prime}}\right)_{\sigma}^{C}=\sqrt{[C]} \sum_{\sigma^{\prime} \sigma^{\prime \prime}} F\left(\begin{array}{l}C^{\prime} C^{\prime \prime} C \\ \sigma^{\prime} \sigma^{\prime \prime} \sigma\end{array}\right) T_{\sigma^{\prime}}^{C^{\prime}} T_{\sigma^{\prime \prime}}^{C^{\prime \prime}}$

where $C, C^{\prime}, C^{\prime \prime}$ are irreducible representations, $\sigma, \sigma^{\prime}, \sigma^{\prime \prime}$ are their rows, and $F\left(\begin{array}{l}C^{\prime} C^{\prime \prime} C \\ \sigma^{\prime} \sigma^{\prime \prime} \sigma\end{array}\right)$ are $3 G$ symbols corresponding to Clebsch-Gordan coefficients of the $C_{3 \mathrm{v}}$ symmetry group. Here [C] stands for the dimension of $C$. The recoupling scheme used for calculations of matrix elements is similar to one described in ref. [4ㄹ] $]$. Using the standard definition of the reduced matrix elements $[\underline{41}, 4 \underline{2}]:$

$$
\left\langle\psi^{\prime\left(C^{\prime}\right)}\left\|V^{(C)}\right\| \psi^{\prime \prime\left(C^{\prime \prime}\right)}\right\rangle=\frac{\left\langle\psi_{\sigma^{\prime}}^{\prime\left(C^{\prime}\right)}\left\|V_{\sigma}^{(C)}\right\| \psi_{\sigma^{\prime \prime}}^{\prime\left(C^{\prime \prime}\right)}\right\rangle}{F\left(\begin{array}{lll}
C^{\prime} & C & C^{\prime \prime} \\
\sigma^{\prime} & \sigma & \sigma^{\prime \prime}
\end{array}\right)}
$$

and the recoupling matrix elements formula:

$$
\begin{aligned}
& \left\langle\left(\psi_{1}^{C_{1}^{\prime}} \psi_{2}^{C_{2}^{\prime}}\right)^{C^{\prime}}\left\|\left(V_{1}^{\Gamma_{1}} V_{2}^{\Gamma_{2}}\right)^{\Gamma}\right\|\left(\psi_{1}^{C_{1}^{\prime \prime}} \psi_{2}^{C_{2}^{\prime \prime}}\right)^{C^{\prime \prime}}\right\rangle= \\
& \sqrt{\left[C^{\prime}\right][\Gamma]\left[C^{\prime \prime}\right]}\left(\begin{array}{ccc}
C_{1}^{\prime} & C_{2}^{\prime} & C^{\prime} \\
\Gamma_{1} & \Gamma_{2} & \Gamma \\
C_{1}^{\prime \prime} & C_{2}^{\prime \prime} & C^{\prime \prime}
\end{array}\right)\left\langle\psi_{1}^{C_{1}^{\prime}}\left\|V_{1}^{\Gamma_{1}}\right\| \psi_{1}^{C_{1}^{\prime \prime}}\right\rangle\left\langle\psi_{2}^{C_{2}^{\prime}}\left\|V_{2}^{\Gamma_{2}}\right\| \psi_{2}^{C_{2}^{\prime \prime}}\right\rangle,
\end{aligned}
$$

where the indices $C$ and $\Gamma$ denote point group irreducible representations (all other indices are omitted for the sake of simplicity) a multi-dimensional matrix element can be expressed as a sum of the one-dimensional matrix elements products.

The use of a nonorthogonal angular basis does not complicate the procedure for obtaining eigenvalues. There are two methods of solution eigenvalue vibrational problem. In first method, one can solve the eigenvalue angular problem, and then use the resulting orthogonal eigenvectors to construct the symmetrized orthogonal angular basis. In second method, one can use a nonorthogonal vibrational basis set. To solve the symmetric-definite generalized eigenvalue problem, standard computational programs, such as programs available in LAPACK library can be applied. Note that this technique does not consume too much additional main memory because 


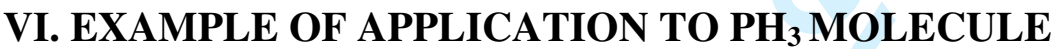

In the present work, we employ potential in the mass-dependent orthogonal coordinates that has been determined in the paper Ref. [25]. One-dimensional eigenfunctions were computed from the multi-dimensional PES by fixing all other coordinates to the equilibrium values. For the stretching coordinates, the following Morse type function was used:

$$
f\left(r^{\prime} ; a\right)=1-\exp \left[-a\left(r_{i}^{\prime}-r_{e}^{\prime}\right)\right]
$$

where $a=1.9$. This value of the $a$ parameter ensures that the second order term of the potential provides a reliable representation for the one-dimensional stretching cut. The terms of higher orders result in relatively small corrections. For the interbond angular coordinates, the following functions were used:

$$
\phi\left(q_{i j}\right)=\cos \left(q_{i j}\right)-\cos \left(q_{\mathrm{e}}\right)
$$


The above defined elementary functions (20-21) were used to build six symmetrized linear combinations of $S R_{A_{1}}, S R_{E a}, S R_{E b}, S A_{A_{1}}, S A_{E_{a}}, S A_{E_{b}}[24,25]$ by using three projection operators $P_{A_{1}}, P_{1 E a}, P_{1 E b}$ from six existing projection operators (17) for the group of permutations of three $\mathrm{H}$-atoms. The subindices correspond to irreducible representations of the $C_{3 \mathrm{~V}}$ point group. In order to determine the six-dimensional (6D) expansion terms of the $A_{1}$ symmetry type, we applied the two step procedure described above. First step corresponds to the construction of the symmetrized powers, and second step to the coupling of the symmetrized powers of different symmetrized coordinates in irreducible trees. A set of all possible trees of the $A_{1}$ representation gives a final set of the $6 D$ expansion terms. Two types of localized one-dimensional wave functions were constructed: $Q_{n}\left(q_{i j}\right)$ - functions of bending angles $q_{i j}$, and $R_{n}\left(r_{i}\right)$ - functions of radial coordinates $r_{i}$. One can obtain the symmetrized vibrational basis set by coupling the $\left|n_{12} n_{13} n_{23}, G_{A} \sigma_{A}\right\rangle$ and $\left|n_{1} n_{2} n_{3}, G_{R} \sigma_{R}\right\rangle$ basis functions using Clebsch-Gordan coefficients of the $C_{3 \mathrm{v}}$ symmetry group.

To ensure a rapid computation of the irreducible matrix elements, we applied the following technique. We first calculate all $\mathrm{J}$-coefficients $(6,8,12,15)$. After this, we calculate angular and radial three-dimensional matrix elements and store results into main memory. It is not necessary to use an optimization of calculations to get the radial matrix elements. At the same time, one needs to use an optimization of calculations to get the angular irreducible matrix elements.

Several convergence tests involving calculated vibration levels have been already performed using various analytical PES representations and $C_{2 v}$ symmetry [25]. A good agreement between vibration levels calculated using two PES fitted in orthogonal massdependent coordinates (10) and in internal mass-independent coordinates have been already reported in Ref. [25]. In this section, we check only the full symmetry nonorthogonal vibrational basis set convergence in orthogonal mass-dependent coordinates (10). Calculations with 
increasing dimensions for the basis cut-off suggest that our vibration energies up to $7000 \mathrm{~cm}^{-1}$ are converged in average to $0.01 \mathrm{~cm}^{-1}$ or better. The corresponding residuals increase gradually with energies. Similarly to Ref. [25], in the present paper, we use the nonsymmetrized vibrational basis dimension as the basis dimension. All calculations have been performed using a full symmetry-adapted set of basis functions. The used basis functions have been grouped into the $\mathrm{A}_{1}$, $\mathrm{A}_{2}$, and $\mathrm{E}$ symmetry classes. Let us consider four basis sets referred to as I, II. III, IV. The dimensions of those basis sets are 14643, 16047, 17889, and 19230 functions, respectively. The

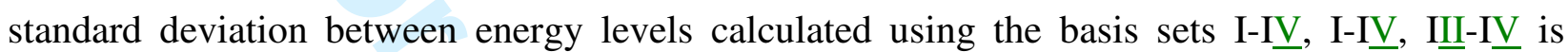
$0.034482,0.002616$, and $0.000571 \mathrm{~cm}^{-1}$, respectively, up to $7000 \mathrm{~cm}^{-1}$. Because all lower calculated energy levels show a slight difference with respect to those obtained in Ref. [25] only four observed band centers in the region $6700-7000 \mathrm{~cm}^{-1}$ were considered. Comparison of these observed band centres of $\mathrm{PH}_{3}$ molecule and calculated from PES[25] in four basis sets I, II, III, IV given in Table 1 . The deviations between vibration levels calculated using vibrational basis set IV and basis sets I, II, and III are shown in Fig. 2. Similarly to the results of calculations using internal coordinates [25], the main contribution to this standard deviation results from two levels above $6500 \mathrm{~cm}^{-1}$ shifted to 0.3 and $0.5 \mathrm{~cm}^{-1}$ (these levels are not shown in Fig. 2) and without these levels the standard deviation between all set is less $0.01 \mathrm{~cm}^{-1}$. It is possible, that the considerable deviation for these two levels is resulted from wrong asymptotic behavior of the potential when the molecule is far from its equilibrium geometry. In general, one can come to the conclusion that calculations using the nonorthogonal $C_{3 v}$ basis and the basis reported in the Ref. [25] result in the similar convergence behavior. For example, according to results from Ref. [25], the standard deviation between two vibrational calculations using 12000 and 15000 nonsymmetrised basis functions is $0.048 \mathrm{~cm}^{-1}$. At the same time, the use of the full symmetry provides that the computation speed of vibration levels is several times faster than for 
computations using the $C_{2 v}$ basis. In addition, the computations using the full symmetry require less memory space that allows us to work with big basis sets.

\section{DISCUSSION AND CONCLUSION}

The primary motivation for this study was to demonstrate a good convergence of new angular basis and to check algorithm of the matrix elements calculation. To do this, we dealt only with a solution of vibrational problem using orthogonal coordinates. At the same time, one can calculate the matrix elements of the vibration-rotation and nonorthogonal terms of the Hamiltonian in a similar way. For example, the matrix elements (14) have been calculated in polar coordinates. The functions of the torsion angle used to write vibration-rotation and nonorthogonal terms of the Hamiltonian can be expressed in terms of three angles $\mathrm{q}_{\mathrm{ij}}$. This allows one to obtain a symmetrical form similar to (11). In addition, the dependence of the PES functions used in Ref. [17] on $\sin \left(\mathrm{q}_{\mathrm{ij}}\right)$ presents no essential difficulties.

The form of angular basis set based on $\cos \left(\mathrm{q}_{\mathrm{ij}}\right)$ product removes the $\sin (\mathrm{q})^{-2}$ singularity from the vibrational kinetic energy operator in polar coordinates [35] also for $\mathrm{AB}_{4}, \mathrm{ABC}_{3}$ type molecules. However, the matrix elements calculations are complicated by the presence of a redundancy among the six angles $\mathrm{q}_{\mathrm{ij}}[19,10]$ for these molecules. On the other hand, symmetrisation of these basis functions can be achieved easily via projector operators technique. For the $\mathrm{CH}_{4}$ molecule, the study of spectra in the range higher than $5400 \mathrm{~cm}^{-1}$ is of considerable importance and it is difficult to obtain a good convergence of the calculated high energy levels without using the $T_{d}$ symmetry. The paper $[8,9,26]$ uses the $C_{3 v}$ symmetry. This means that only 6 from 24 permutations in $T_{d}$ point group are in use. In paper [22], only 8 from 24 elements of $T_{d}$ point group were used. Thus, the cited papers do not employ the full symmetry of the molecule. Of course, one can try to perform a numerical symmetrization of the angular basis, but it is a 


\section{Acknowledgement}

The support of ANR(France) through Grant No. ANR-08-BLAN-0254-04 is acknowledged. The support of the Groupement de Recherche International SAMIA between CNRS (France), RFBR (Russia) and CAS (China) is acknowledged. We acknowledge the support from the computer centre Reims-Champagne-Ardenne and the computer centre SKIF Syberia of Tomsk. The support of RFBR(Russia) through Grants 09-05-92508 and 09-09-93103 is acknowledged. The support of CRDF(USA) through Grant RUG1- 2954-TO-09 is also acknowledged. 


\section{References}

[1] H. Partidge, D.W. Schwenke J. Chem. Phys. 106, 4618, (1996)

[2] O. L. Polyansky, A. G. Császár, S. V. Shirin, N. F. Zobov, P. Barletta, J. Tennyson, D. W. Schwenke, and P. J. Knowles, Science 299, 539 (2003)

[3] P. Barletta, S.V. Shirin, N.F.Zobov, O.L.Polyansky, J. Tennyson, E.F.Valeev, A.G. Csaszar, J. Chem. Phys. 125, 204307 (2006)

[4] D.W. Schwenke, H. Partidge, Spectrochimica Acta Part A. 57, 887 (2001)

[5] D.W.Schwenke, Spectochem. Acta Part A. 58, 849 (2002)

[6] H.G.Yu,J.Chem.Phys.120, 2270 (2002).

[7] S. Carter, H. M. Shnider, and J. M. Bowman, J. Chem. Phys. 110, 8417 (1999).

[8] X.G.Wang and T.Carrington,J.Chem.Phys.119, 101 (2003).

[9] X.G.Wang and T.Carrington,J.Chem.Phys.118, 6260 (2003).

[10] S. A. Manson and M. M. Law, Phys. Chem. Chem. Phys., 8, 2848 (2006).

[11] S. A. Manson, M. M. Law, I. A. Atkinson and G. A. Thomson, Phys. Chem. Chem. Phys., 8, 2855 (2006).

[12] J. Makarewicz and A. Skalozub, Spectrochimica Acta Part A 58, 601 (2002).

[13] S. N. Yurchenko, W. Thiel, S. Patchkovskii and P. Jensen, Phys. Chem. Chem. Phys. 7, 573 (2005).

[14] S. N. Yurchenko, M. Carvajal, P. Jensen, F. Herregodts and T.R. Huet, Chem. Phys. 290, 59 (2003).

[15] H.G.Yu,J.Chem.Phys.117, 2030 (2002).

[16] H.S.Leeand, J.C.Light,J.Chem.Phys.118, 3458 (2003).

[17] R. Ovsyannikov, S. N. Yurchenko, M. Carvajal, W. Thiel and P. Jensen, J. Chem. Phys. 129, 044309 (2008).

[18] X.G.Wang, E.L. Sibert III, Spectrochimica Acta Part A. 58, 863-872 (2002)

[19] L.Halonen,J.Chem.Phys.106, 831 (1995). 
[20] N. C. Handy, S. Carter, and S. M. Colwell, Mol. Phys. 96, 477 (1999)

[21] J. M. Bowman, T. Carrington, and H.-D. Meyer, Molecular Physics, 106,2145 (2008)

[22] D.W.Schwenke, J.Chem.Phys.118, 10431 (2003).

[23] B. Poirier, J. Chem. Phys. 119, 90 (2003).

[24] M. Rey, A.V. Nikitin and Vl.G. Tyuterev, Molecular Physics,108,2257 (2010)

[25] A. V. Nikitin, F. Holka, V. G. Tyuterev, and J. Fremont J.Chem. Phys., 130, 244312 (2009)

[26] A. V. Nikitin, S. Mikhailenko, I. Morino, T. Yokota, R. Kumazawa and T. Watanabe, JQSRT, 110, 964 (2009)

[27] A. V. Nikitin., J. Mol. Spectrosc. 252, 17 (2008).

[28] I.N. Kozin, M.M. Law, J.M. Hutson, J. Tennyson, J. Chem. Phys. 118, 4896 (2003)

[29] I.N. Kozin, J. Tennyson, M.M. Law, Computer Physics Communication 165, 10 (2005)

[30] J. Xie, J.Tennyson, Molecular Physics, 100, 1615 (2002)

[31] J. Xie, J.Tennyson, Molecular Physics, 100, 1623 (2002)

[32] J.Tennyson and B.T.Sutcliffe, J.Chem.Phys., 77, 4061(1982).

[33] F. Gatti, C. Iung, M. Menou, Y. Justum, A. Nauts, X. Chapuisat. J.Chem.Phys., 108, 8804 (1998)

[34] F. Gatti, C. Iung, M. Menou, Y. Justum, A. Nauts, X. Chapuisat. J.Chem.Phys., 108, 8821 (1998)

[35] M. Mladenović, J.Chem. Phys. 112, 1070 (2000).

[3ㅁ] M. Mladenović, J.Chem. Phys. 112, 1082 (2000).

[37] A. V. Nikitin, Opt. Atmosphere and ocean, 15, 722 (2002)

[3ㄹ] M. Mladenovic, Molecular Physics, 101, 3527 (2002)

[39] S. K. Pogrebnya, A.J. Richardson, D.C. Clary, Chemical Physics Letters, 346, 149, (2001).

[40] D.A.Varshalovich, A.N. Moskalev, V.K. Khersonskii, Quantum theory of Angular momentum, World Scientific, 2008 
[41] B. I. Zhilinskii, V. I. Perevalov and V1. G. Tyuterev, Method of Irreducible Tensorial Operators in the Theory of Molecular Spectra, (Nauka, Novosibirsk, 1987).

[42] J. P. Champion, M. Loete and G. Pierre, Spherical top spectra in Spectroscopy of the Earth's Atmosphere and Interstellar Medium, (Academic Press, Boston, 1992).

[4ㄹ] A. Nikitin, J. P. Champion and Vl. G. Tyuterev, J. Mol. Spectrosc. 182, 72 (1997).

[44] S.-G. He, J. J. Zheng, S.-M. Hu, H. Lin, Y. Ding, X.-H. Wang, and Q.-S.

Zhu, J. Chem. Phys. 114, 7018 (2001)

[4ㄷ] L. Wei , Computer Physics Communications, 120, 222 (1999)

[4ㅌ] T.A. Heim, J. Hinze and A. R. Rau J.Phys A: Math Theor 42, 175203 (2009) 


\section{Figure captions}

\section{Figure 1}

Binary tree of vibrational coupling for $\mathrm{AB}_{3}$ molecules. In the left part: tree of the PES parameters. In the right part: tree of the basis function coupling.

\section{Figure 2}

Deviations between vibration levels of $\mathrm{PH}_{3}$ molecule calculated in various vibrational basis sets.

Table I. Comparison of some observed band centers of $\mathrm{PH}_{3}$ and calculated from PES[25] in four basis sets I, II, III, IV. All values are in $\mathrm{cm}^{-1}$.

\begin{tabular}{|l|l|l|l|l|l|}
\hline $\mathrm{PH}_{3}$ band & Obs. $\dagger$ & I & II & III & IV \\
\hline $3 v_{3}(\mathrm{E})$ & 6714.60 & 6714.769 & 6714.771 & 6714.766 & 6714.766 \\
\hline $2 v_{1}+v_{3}(\mathrm{E})$ & 6883.73 & 6886.345 & 6886.348 & 6886.344 & 6886.344 \\
\hline$v_{1}+2 v_{3}(\mathrm{E})$ & 6890.86 & 6890.122 & 6890.125 & 6890.121 & 6890.120 \\
\hline $3 v_{3}\left(\mathrm{~A}_{1}\right)$ & 6971.16 & 6971.360 & 6971.362 & 6971.359 & 6971.358 \\
\hline
\end{tabular}

$\dagger$ See Ref $[4 \underline{4}]$ 


\title{
Special characters
}

\author{
$\dagger$ footnote \\ $v \quad$ lower case nu
}


Binary tree of vibrational coupling for AB3 molecules. In the left part: tree of the PES parameters. In the right part: tree of the basis function coupling. $215 \times 279 \mathrm{~mm}(600 \times 600 \mathrm{DPI})$ 


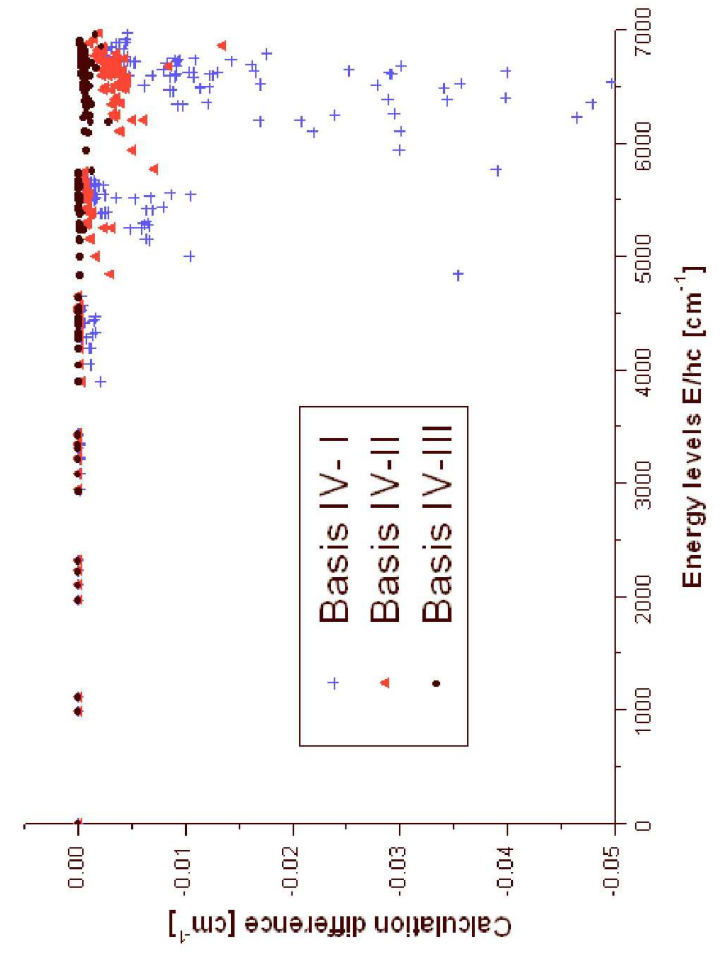

Deviations between vibration levels of PH3 molecule calculated in various vibrational basis sets. $215 \times 279 \mathrm{~mm}(600 \times 600 \mathrm{DPI})$ 
Reply to the Reviewer 1 (R1) of the manuscript TMPH-2010-0349

by A.V. Nikitin

In the Introduction (and possibly later) the reference list [4-27] might include the papers of Kozin et al (2003-5) which also consider and apply some of the topics considered in this paper (orthogonal coordinates, contracted angular basis functions, efficient calculation of matrix elements ...) in the context of 4-atomic molecules.

Reply $>$ I added two references [28,29]

Also in the first paragraph of the Intro, references [34,35] could be cited in the context of the symmetry of $\mathrm{AB} 4$ systems.

Reply> I added next sentence to the introduction: One variational method for calculating excited bending states of symmetric tetrahedral pentaatomic molecules based on the use of Radau coordinates and Jacobi polynomials as the basis functions has been presented in $[30,31]$.

p4, line 43, which "New Algorithm"? The one to be described in This Work?

Reply> Replaced on "Described in this work algorithm

p5, line 54, has "t23" been defined?

Reply> I added the definition

p7, line 8, insert "angular" before "PES"

Reply> OK

p7, line 8 , should "the power functions" read "a sum of product functions"?

Reply> OK

p8 (and elsewhere), I think the symbol $\mathrm{q}$ is being used in too many different and confusing ways: general coordinates with 1 subscript (as in equation 11) and also interbond angles with 2 subscripts (as in line 3) or no subscripts (as in line 36).

Reply> OK, general coordinates changed to $\mathrm{R}$

p8, line 48, should "couple" be just "pair"?

Reply $>$ OK

p9, line 13, what are "linear in q_ij terms"?

Reply> Changed to 'first derivative

p9, why has "cos(q_ij)" in line 18 become just "q_ij" in line 45?

Reply> My misprint! Line 18 changed to q 
p9, line 29, what are the "reduced matrix elements"? (They are defined later, but I think need some explanation here or earlier).

Reply> I just added two references because there are explanation in next paragraph

p11, line 3, "The not symmetry-adapted" should be "The initial" (and I think everything after "symmetrised" could be deleted in that sentence!).

Reply> OK, changed

p11, line 21, there are 2 closing brackets missing in the first formula.

Reply> OK, changed

p11 \& fig 1, the "binary tree" seems trivial - maybe all is explained in ref [41] but perhaps a further sentence of explanation here would make the significance of this clearer?

Reply> I agree that figure 1 is trivial. But readers very often don't understand coupling scheme. I added some details in the text. Figure 1 is also updated.

p11, line 46, "the symmetrized coordinates $\mathrm{Si}$ " (as distinct from symmetrised basis functions) appear suddenly and without definition - again some explanation might help the reader.

Reply> Si replaced and text is changed

p13, line 3, "In the sequel" is not clear (does it mean "next" or "in practice" ...?)

Reply> Text changed

p15, lines 20-27, apparently a sentence is repeated but with different numbers!

Reply> Deleted one sentence

p15 lines 27-9 and Table 1, why only compare with 4 observed band centres? What makes these special? Are there no other observations?

Also, how do the calculated vibrational levels compare with other variational calculations on this PES?

Reply> I added one sentence to text. Calculated levels are very closed to [25]. The purpose of article just demonstrate that the algorithm work. Orthogonal and non orthogonal surfaces [25] are a little different so direct comparing is not informative.

p15, the speculation about "some peculiarities of the potential" seems very vague - can the author be more specific? Can he say at least how the two large deviations vary with basis set? 
Reply> Potential don't adapted for calculation of high exited states $(>7000 \mathrm{~cm}-1)$. Wrong asymptotic behavior of PES leads to problems with basis convergence. New PES is under construction now.

Other minor errors (but NOT an exhaustive list!):

p1, in the title, "of energy levels calculation for the AB3 types molecules" should be "for the calculation of energy levels of AB3 type molecules"

Reply> OK, changed

All through the text the author has trouble with articles ("the" and "a"); for example on p2, line 6, "the symmetry-adapted" should be "a symmetry-adapted"; line 10, insert "the" before "kinetic" and line 20, insert "the" before "PH3".

Reply> Changed

p2, line 15, "vibrational matrix elements calculation" should be "the calculation of vibrational matrix elements".

Reply> OK, changed

p3, line 24, should "numbers" be "quantum numbers" or "atoms"?

Reply> OK, changed to atoms

p4, line 29, "has no the" should be "does not have the" or "has no"

Reply> OK, changed to has no

p4, line 43, "not orthogonal" should be "non-orthogonal"

Reply> OK, changed

p4, line 53, "completion" should be "final"

Reply> OK, changed

p5, line 39, insert "of" after "form"

Reply> OK, changed

p6, line 55, Should "In a particular case" be "In three particular cases" or "For example"? Reply> OK, changed to for example

p7, line 11, the notation " $n \_i j "$ is used for the powers of the cos functions but it seems to be $P_{-}$ij thereafter.

Reply> OK, changed

p11, line 51, "obtained" should be "resulting"

Reply> OK, changed

p15, line 31, delete "standard" 
Reply> OK, changed

p15 line 22, "CM" should be lower case.

Reply> OK, changed

p16, line 13, "approve" should be "demonstrate"

Reply> OK, changed

p17, line 40, "prominent" should be "important.

Reply> OK, changed

$\mathrm{p} 21$, footnote to Table 1, why not give the original references here?

Reply $>$ OK, added 